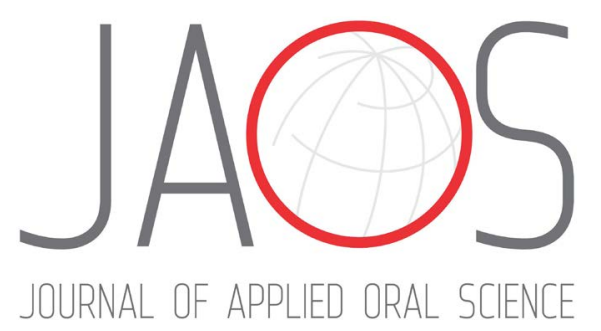

\title{
Role of extracellular DNA in Enterococcus faecalis biofilm formation and its susceptibility to sodium hypochlorite
}

\section{Abstract}

Mi-Kyung $\mathrm{YU}^{1,2,3}$ iD

Mi-Ah KIM ${ }^{1}$

Vinicius ROSA ${ }^{4}$

Yun-Chan HWANG ${ }^{5}$

Massimo DEL FABBRO ${ }^{6,7}$

Won-Jun SOHN ${ }^{8}$

Kyung-San MIN ${ }^{1,2,3}$ iD
Submitted: November 15, 2018

Modification: March 14, 2019 Accepted: March 25, 2019

Corresponding address: Kyung-San Min

Department of Conservative Dentistry School of Dentistry and Institute of Oral Bioscience, Chonbuk National University. 567 Baekje-daero, Deokjin-gu, Jeonju-si, Jeollabuk-do
-54896 - Republic of Korea.
Phone - 82-63-250-2764 - FAX: 82-63-250-2129567 Baekje-daero, Deokjin-gu, Jeonju-si, Jeollabuk-do
-54896 - Republic of Korea.
Phone - 82-63-250-2764 - FAX: 82-63-250-2129e-mail: endomin@gmail.com
Objective: This study investigated the role of extracellular deoxyribonucleic acid (eDNA) on Enterococcus faecalis ( $E$. faecalis) biofilm and the susceptibility of $E$. faecalis to sodium hypochlorite $(\mathrm{NaOCl})$. Methodology: $E$. faecalis biofilm was formed in bovine tooth specimens and the biofilm was cultured with or without deoxyribonuclease (DNase), an inhibitor of eDNA. Then, the role of eDNA in $E$. faecalis growth and biofilm formation was investigated using colony forming unit (CFUs) counting, eDNA level assay, crystal violet staining, confocal laser scanning microscopy, and scanning electron microscopy. The susceptibility of $E$. faecalis biofilm to low $(0.5 \%)$ or high $(5 \%) \mathrm{NaOCl}$ concentrations was also analyzed by CFU counting. Results: CFUs and biofilm formation decreased significantly with DNase treatment $(p<0.05)$. The microstructure of DNase-treated biofilms exhibited less structured features when compared to the control. The volume of exopolysaccharides in the DNase-treated biofilm was significantly lower than that of control $(p<0.05)$. Moreover, the CFUs, eDNA level, biofilm formation, and exopolysaccharides volume were lower when the biofilm was treated with DNase de novo when compared to when DNase was applied to matured biofilm $(p<0.05)$. E. faecalis in the biofilm was more susceptible to $\mathrm{NaOCl}$ when it was cultured with DNase $(p<0.05)$. Furthermore, $0.5 \% \mathrm{NaOCl}$ combined with DNase treatment was as efficient as $5 \% \mathrm{NaOCl}$ alone regarding susceptibility $(p>0.05)$. Conclusions: Inhibition of eDNA leads to decrease of $E$. faecalis biofilm formation and increase of susceptibility of $E$. faecalis to $\mathrm{NaOCl}$ even at low concentrations. Therefore, our results suggest that inhibition of eDNA would be beneficial in facilitating the efficacy of $\mathrm{NaOCl}$ and reducing its concentration.

Keywords: DNA. Enterococcus faecalis. Sodium hypochlorite. Biofilms.

${ }^{1}$ Chonbuk National University, School of Dentistry and Institute of Oral Bioscience, Department of Conservative Dentistry, Jeonju, Korea.

${ }^{2}$ Chonbuk National University, Research Institute of Clinical Medicine, Jeonju, Korea.

${ }^{3}$ Chonbuk National University Hospital, Biomedical Research Institute, Jeonju, Korea.

${ }^{4}$ National University of Singapore, Faculty of Dentistry, Discipline of Oral Sciences, Singapore.

${ }^{5}$ Chonnam National University, School of Dentistry, Department of Conservative Dentistry, Gwangju, Korea.

${ }^{6}$ Università degli Studi di Milano, Dipartimento di scienze biomediche, chirurgiche e odontoiatriche, Milan, Italy.

${ }^{7}$ RRCCS Istituto Ortopedico Galeazzi, Milan, Italy

${ }^{8}$ Seoul National University, Dental Research Institute and School of Dentistry, Department of

Conservative Dentistry, Seoul, Korea. 


\section{Introduction}

Enterococcus faecalis (E. faecalis) is a bacterium frequently recovered from infected root canal systems. ${ }^{1,2}$ This bacteria is difficult to remove since it is able to form biofilms and survive under a wide range of acidic and basic conditions and prolonged periods of nutritional deprivation. ${ }^{3,4}$ E. faecalis biofilms consist of exopolysaccharides, proteins, lipids, and extracellular deoxyribonucleic acid (eDNA).5,6 The dense and protected environment of a biofilm may facilitate gene transfer and enhance biofilm stability. ${ }^{7}$ As a major structural component of many different microbial biofilms, the importance of eDNA was first reported in Pseudomonas aeruginosa. ${ }^{5}$ The eDNA is released via autolysis in a fratricidal or suicidal manner and/or active release through membrane vesicles and nanofibers in E. faecalis biofilms. ${ }^{6,8}$ Previous reports have attributed a crucial role to eDNA in the formation, mechanical stability, and maturation of bacterial biofilms in general and $E$. faecalis biofilms in particular. ${ }^{9-11}$ Irrigation is critical to remove microorganisms from root canal systems. $\mathrm{NaOCl}$ is the most frequently used material for endodontic treatment, it is an antiseptic and inexpensive lubricant that has been used at dilutions ranging from $0.5 \%$ to $5.25 \% .{ }^{12}$ Usually, it is assumed that a higher concentration of $\mathrm{NaOCl}$ increases the efficacy in removing bacteria within root canal systems; however, severe complications of $\mathrm{NaOCl}$ extrusion during endodontic treatment can occur at high concentrations. ${ }^{13} \mathrm{NaOCl}$ is a strong oxidizing agent and may cause significant damage when in direct contact with tissue, including rapid hemolysis and ulceration, inhibition of neutrophil migration, and destruction of endothelial and fibroblast cells. ${ }^{14}$ Therefore, there have been several alternative approaches proposed to improve the effectiveness of lower-concentration $\mathrm{NaOCl}$ solutions to avoid extensive tissue damage; however, these options have many limitations, including the requirement of inconvenient equipment. ${ }^{15-19}$ Since eDNA is an essential component of $E$. faecalis biofilm, it can be speculated that its inhibition using a simple agent can be another strategy for effective biofilm removal. However, to our knowledge, there has been little information regarding the effect of eDNA on $E$. faecalis biofilm in endodontic study models. Therefore, the aim of this study was to investigate the role of eDNA in the formation of $E$. faecalis biofilm in bovine root canal systems through
DNase, a known eDNA inhibitor. Moreover, this study highlights the susceptibility of the bacteria to $\mathrm{NaOCl}$ by eliminating eDNA in the biofilm.

\section{Methodology}

\section{Preparation of specimens}

This study used recently extracted single-rooted bovine central incisors. The teeth were immersed in $1 \% \mathrm{NaOCl}$ solution for $24 \mathrm{~h}$ for surface disinfection. Following, each tooth was horizontally sectioned 5 $\mathrm{mm}$ in length below the cementoenamel junction using a diamond saw (AEU-25, Aseptico, Woodinville, WA, USA) at $15,000 \mathrm{rpm}$. The root canals of the cylindrical specimens were enlarged using a $3.1 \mathrm{~mm}$ diameter round bur. Next, each specimen was vertically sectioned with a diamond saw into cylindrical halves (Figure 1a). Finally, the smear layer was removed using $17 \%$ ethylenediaminetetraacetic acid solution. To prevent bacterial contamination, the specimen was steam-sterilized in an autoclave (LAC-5101SD, DAIHAN Lab Tech, Namyangju, Korea) at $121^{\circ} \mathrm{C}$ for 20 minutes. To ensure dentin contamination only through the main root canal wall, the outer surface of the sectioned specimens was varnished with a double layer of nail polish.

\section{Infection of the specimens and DNase treatment}

E. faecalis (bacterial strain ATCC 29212) was aerobically cultured in sterile brain heart infusion (BHI; Difco Laboratories, Detroit, MI, USA) medium at $37^{\circ} \mathrm{C}$. The $\mathrm{BHI}$ plates contained $1.5 \%$ (wt/vol) of agar (Difco Laboratories). The specimens were placed vertically in a 48-well plate (SPL, Daejeon, Korea), and $E$. faecalis $\left(6 \times 10^{5} \mathrm{CFU} / \mathrm{mL}\right)$ was transferred to each well. Following, the plates were incubated at $37^{\circ} \mathrm{C}$ for 2 days and divided into 3 groups $(n=9)$ : (i) E. faecalis in $\mathrm{BHI}$ (control), (ii) E. faecalis in BHI with DNase (ELPIS, Daejeon, Korea) treatment at $2 \mathrm{U} / \mu \mathrm{l} /$ $\mathrm{ml}$ for $1 \mathrm{~h}$ after 2-day incubation (DNase-1h), and (iii) E. faecalis and DNase $(2 \mathrm{U} / \mu \mathrm{l} / \mathrm{ml})$ in BHI (DNase-2d).

\section{Colony forming unit (CFU) counting and eDNA measurement}

The specimens were washed with sterile water and then transferred into a $1.5 \mathrm{ml}$ tube containing $1 \mathrm{ml}$ of sterile water. Following, the specimens were sonicated to collect eDNA using a sonifier (10 s, two 
times at 20\% energy level) (VCX 130PB; Sonics \& Materials, Newtown, CT, USA). CFU counting was performed by plating serial dilution of an aliquot (0.1 $\mathrm{ml}$ ) of each specimen on BHI agar plates. The rest were centrifuged at $10000 \times \mathrm{g}$ at $4^{\circ} \mathrm{C}$ for $10 \mathrm{~min}$ and the supernatant was filtered. For eDNA measurement, the supernatant was treated with DNA-binding dye, SYBR Green (Invitrogen, Carlsbad, CA, USA). eDNA concentration was measured with excitation at 485 $\mathrm{nm}$ and emission at $535 \mathrm{~nm}$ using a fluorescence microplate reader (HIDEX, Turku, Finland).

\section{Crystal violet staining}

Crystal violet staining was performed to assess the biofilm mass in the specimens. The specimens were washed with sterile water and added $0.1 \%$ crystal violet (Sigma-Aldrich, St. Louis, MO, USA). Following, the specimens were kept at room temperature for 10 min. Next, they were rinsed with sterile water, treated with 30\% acetic acid (Fisher Scientific, Fair Lawn, NJ, USA), and transferred to a 96-well plate. The absorbance at $595 \mathrm{~nm}$ was then measured ( $\mu$ Quant, Biotek Instrument, Winooski, VT, USA).

\section{Confocal laser scanning microscopy (CLSM)}

One $\mu \mathrm{M}$ of Alexa Fluor 647-labeled dextran conjugate (Molecular Probes, Eugene, OR, USA) was added to the $\mathrm{BHI}$ broth with $E$. faecalis $\left(6 \times 10^{5} \mathrm{CFU} /\right.$ $\mathrm{ml}$ ), and then incubated for 2 days at $37^{\circ} \mathrm{C}$. The fluorescence-labeled dextran was incorporated during exopolysaccharide matrix synthesis over the course of biofilm development. After 2 days, $2.5 \mu \mathrm{M}$ SYTO 9 green fluorescent nucleic acid stain (480/500 nm; Molecular Probes) was added and incubated for 30 min to label the bacteria in the biofilms. Following, CLSM imaging was conducted using an LSM 510 META microscope (Carl Zeiss, Jena, Germany) (EC Plan-Neofluar $10 \times / 0.30$ M27). Three independent experiments were performed and seven image stacks ( $512 \times 512$ pixel tagged image file format) were collected per experiment. For the measurement of exopolysaccharides thickness, $512 \times 512$ pixel fields were collected in a stack of seven slices at a 7.86 $\mu \mathrm{m}$ interval. The exopolysaccharides volumes were quantified from the confocal stacks using COMSTAT (www.comstat.dk; Kongens Lyngby, Denmark). ${ }^{20}$

\section{Scanning electron microscopy (SEM)}

The biofilm formed on the specimens was rinsed with phosphate buffered saline (Sigma-Aldrich) and fixed in $2.5 \%$ glutaraldehyde (Sigma-Aldrich) for $2 \mathrm{~h}$ at $4^{\circ} \mathrm{C}$. Following, the specimens were dehydrated in a graded series of ethanol (25-100\%) and air-dried them at room temperature for $1 \mathrm{~h}$. The samples were coated with gold-palladium and observed using SEM (Hitachi, Tokyo, Japan). The images were obtained on a Hitachi SU-70 using BSE detector with $10.0 \mathrm{Kv}$ voltage acceleration.

\section{$\mathrm{NaOCl}$ treatment and CFU counting}

To study the $E$. faecalis susceptibility to $\mathrm{NaOCl}$ in biofilm treated with DNase, the specimens with $E$. faecalis $\left(6 \times 10^{5} \mathrm{CFU} / \mathrm{ml}\right)$ and DNase $(2 \mathrm{U} / \mu \mathrm{l} / \mathrm{ml})$ were cultured for 2 days at $37^{\circ} \mathrm{C}$. After incubation, the biofilms formed on the specimens were treated with $0.5 \%$ or $5 \% \mathrm{NaOCl}$ for $5 \mathrm{~min}$. Next, the specimen was transferred to a $1.5 \mathrm{ml}$ tube that contained $1 \mathrm{ml}$ of sterile water and sonicated to break the biofilms. CFU counting was performed by plating serial dilution of an aliquot $(0.1 \mathrm{ml})$ of each specimen on $\mathrm{BHI}$ agar plates.

\section{Statistical analysis}

All experiments were performed at least three times. Data was analyzed using 1-way analysis of variance, followed by Tukey's test. The data were presented as mean and standard deviation. Statistical significance was considered when $p>0.05$. These analyses were performed with the SPSS software (SPSS $12.0 \mathrm{~K}$ for Windows; SPSS Inc., Chicago, IL, USA).

\section{Results}

\section{Effect of eDNA on E. faecalis biofilm formation and stability}

The DNase-treated groups exhibited significantly fewer CFUs when compared to the control $(p<0.05)$ (Figure 1b). Furthermore, the DNase-2d group showed fewer CFUs when compared to the DNase1h group $(p<0.05)$. eDNA levels collected from the specimens of each group showed that there was also a significant decrease in DNase treated groups $(p<0.05)$ (Figure 1c). Crystal violet staining showed significantly decreased biofilm formation by $13 \%$ (DNase-1h) and 15\% (DNase-2d) when compared to the control $(p<0.05)$ (Figure $1 d)$. Similar to the CFU counting results, there was a significant difference between the two experimental groups $(p<0.05)$. As 
(a)

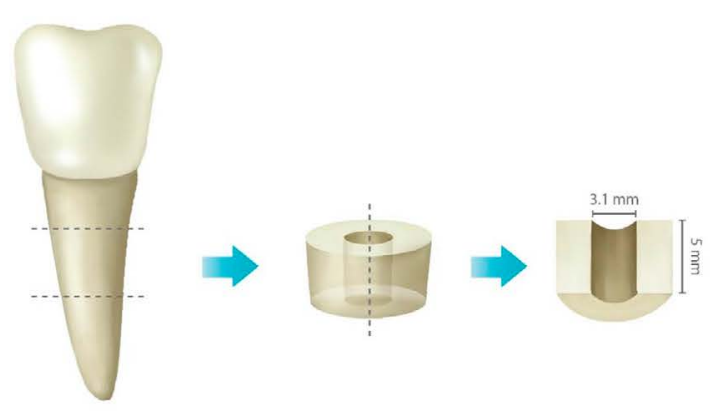

(c)

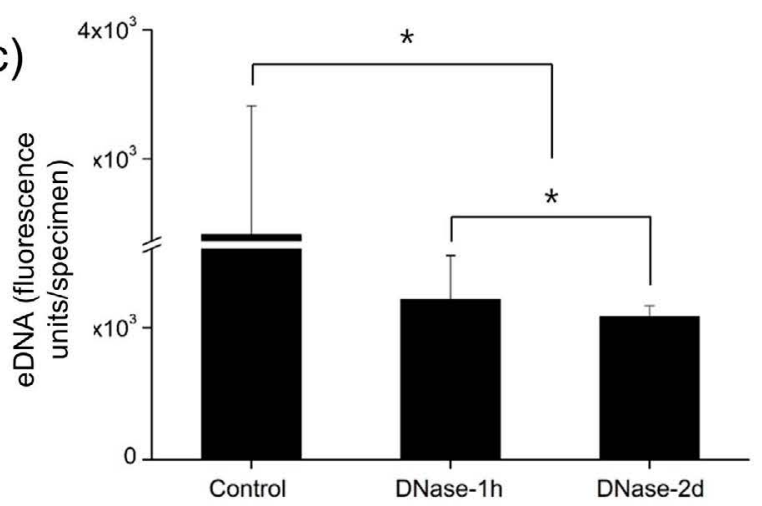

(b)

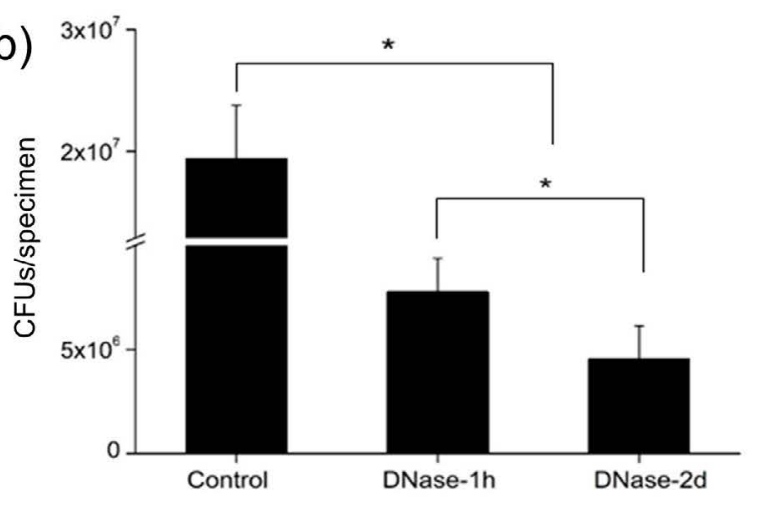

(d)

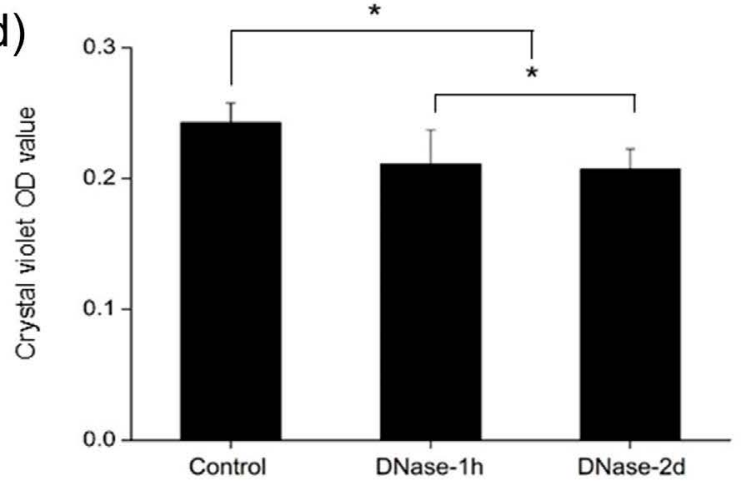

Figure 1- (a) Schematic illustration of the bovine root canal specimen. (b) CFUs of $E$. faecalis biofilms from the specimens. (c) eDNA measured using SYBR green. (d) Biofilm formation measured by crystal violet staining. Control: cultured only in BHI for 2 days, DNase$1 \mathrm{~h}$ : treated with DNase for 1 hour after 2-day culture in BHI, and DNase-2d: cultured with DNase for 2 days. *Statistical significance was determined at $p<0.05$
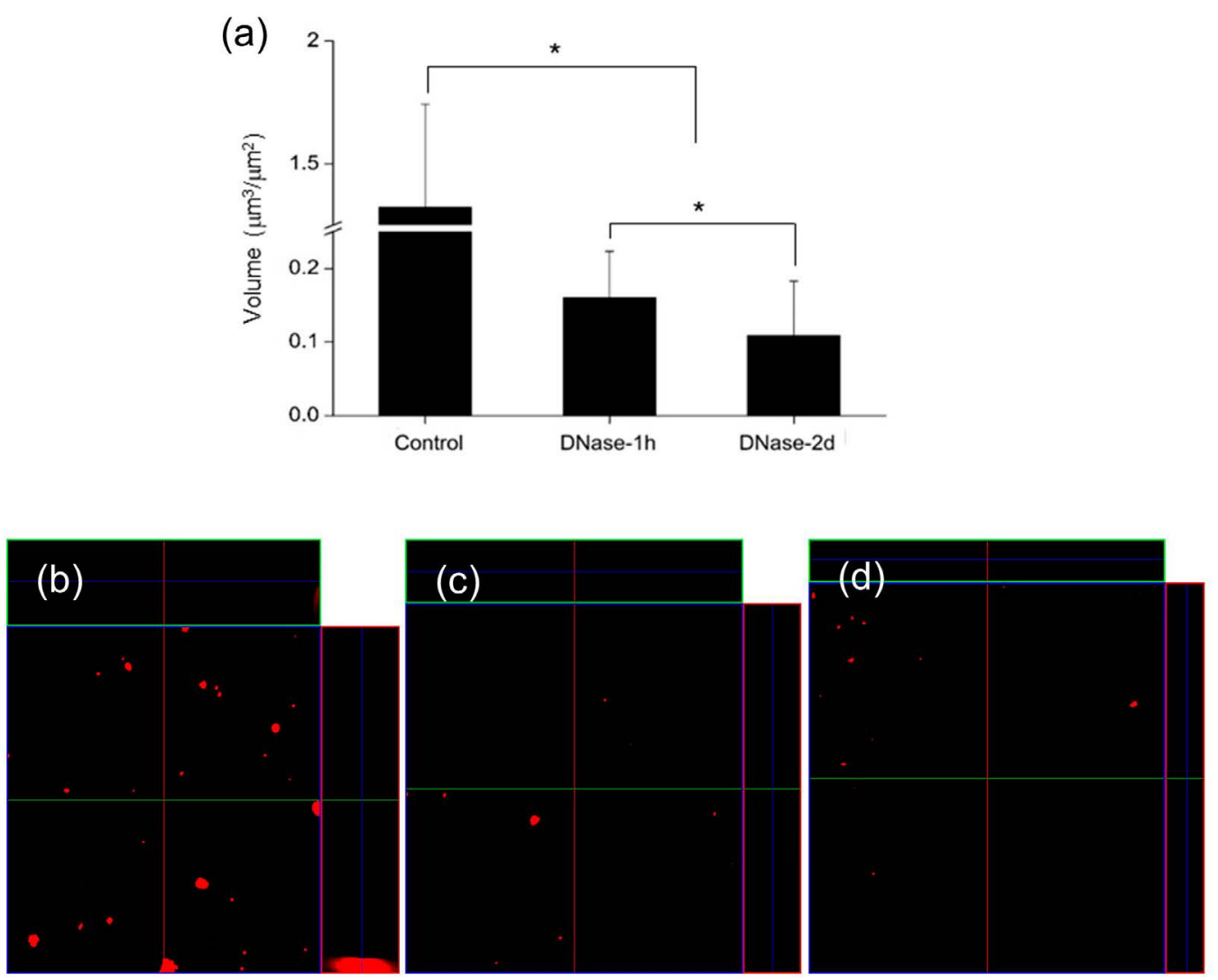

Figure 2- (a) Exopolysaccharides volume in the biofilms. (b-d) Representative CLSM images for measuring exopolysaccharides. Control: cultured only in $\mathrm{BHI}$ for 2 days, DNase-1h: treated with DNase for 1 hour after 2-day culture in $\mathrm{BHI}$, and DNase-2d: cultured with DNase for 2 days. *Statistical significance was determined at $p<0.05$ 
shown in Figure 2a-d, CLSM analysis showed that the exopolysaccharides volume in the DNase-treated biofilm was significantly lower than the control $(p<0.05)$. Moreover, there was a statistical difference between the DNase-1h and DNase-2d groups in terms of exopolysaccharides volume $(p<0.05)$. In SEM observation, DNase-treated biofilms exhibited more porous and disintegrated characteristics when compared to the untreated control (Figure 3).

Effect of DNase on the susceptibility of $E$. faecalis in the biofilm to $\mathrm{NaOCl}$

To investigate whether removal of eDNA from biofilms enhanced the susceptibility of $E$. faecalis to
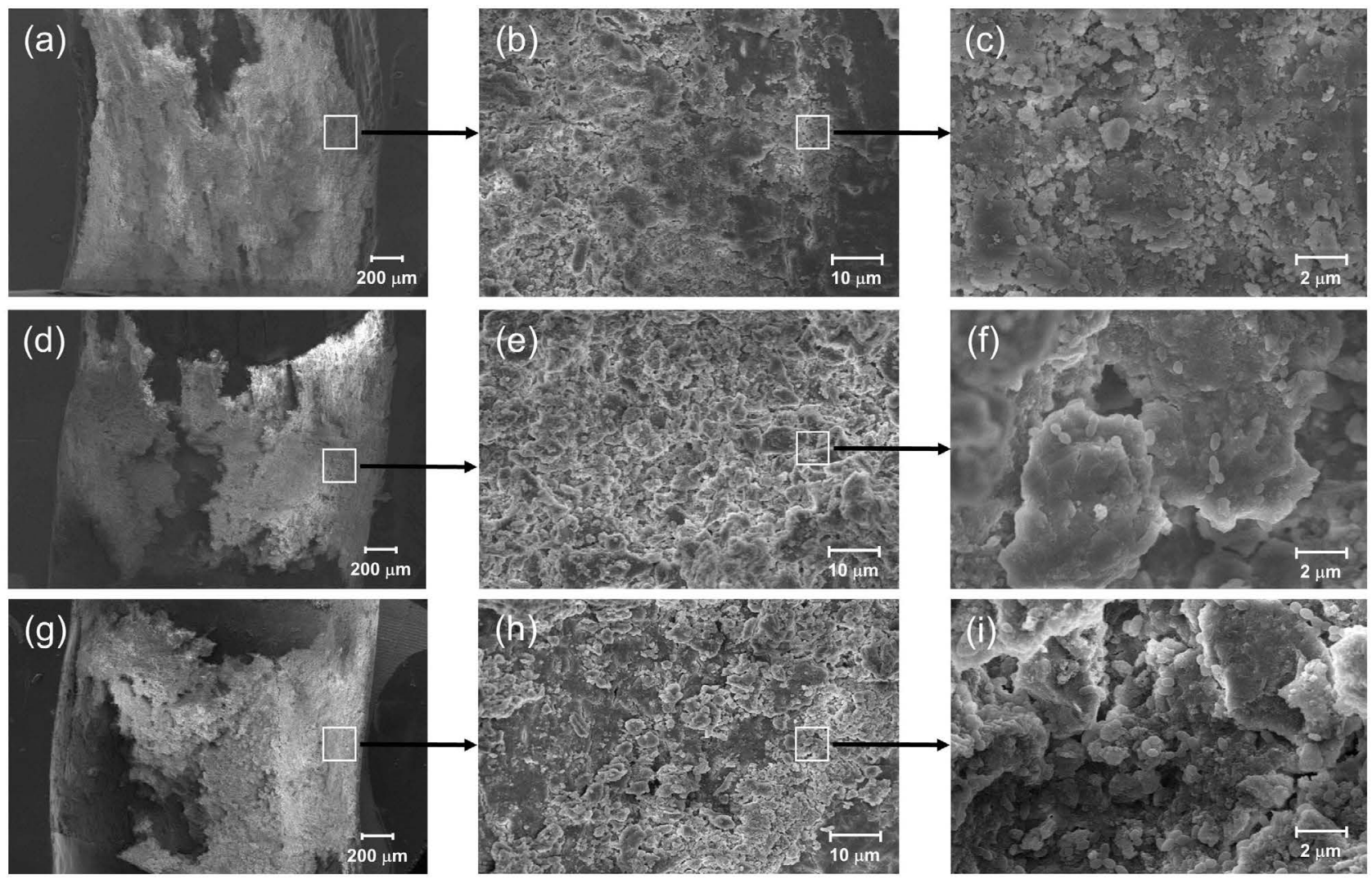

Figure 3-SEM images of $E$. faecalis biofilm formed in the bovine root canal specimens. (a-c): cultured only in $\mathrm{BHI}$ for 2 days (Control), (d-f): treated with DNase for 1 hour after 2-day culture in BHI (DNase-1h), and ( $\mathrm{g}-\mathrm{i})$ : cultured with DNase for 2 days (DNase-2d). Magnification: $(\mathrm{a}, \mathrm{d}$, and $\mathrm{g})-30 \mathrm{X},(\mathrm{b}, \mathrm{e}$, and $\mathrm{h})-1000 \mathrm{X}$, and $(\mathrm{c}, \mathrm{f}$, and $\mathrm{i})-50000 \mathrm{X}$

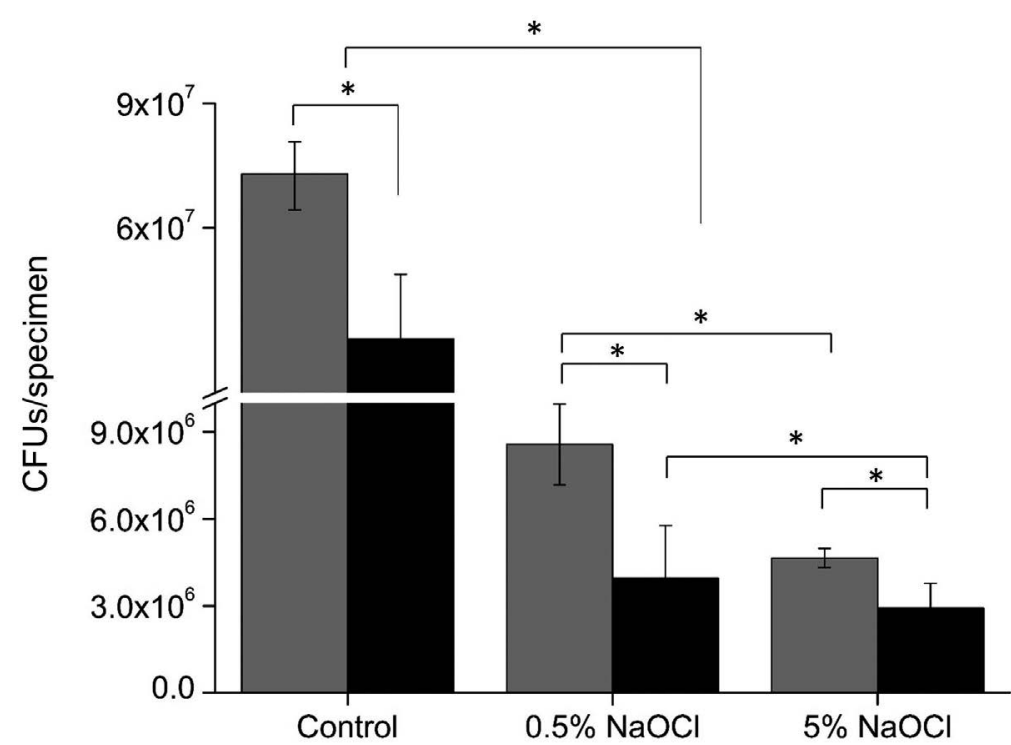

Figure 4- $\mathrm{CFU}$ assay with $\mathrm{NaOCl}$ treatment. E. faecalis were cultured in $\mathrm{BHI}$ without DNase (gray bar) or with DNase (black bar) for 2 days, and then the biofilms were treated with 0.5 or $5 \%$ of $\mathrm{NaOCl}$ for 5 minutes. Control: cultured only in $\mathrm{BHI}$ for 2 days without $\mathrm{NaOCl}$ treatment. ${ }^{*}$ Statistical significance was determined at $p<0.05$ 
$\mathrm{NaOCl}$ within the biofilms, $0.5 \%$ and $5 \% \mathrm{NaOCl}$ were used to treat the biofilms with or without DNase for 2 days. As shown in Figure 4, the biofilms treated with $\mathrm{NaOCl}$ had significantly fewer CFUs when compared to the control that received no $\mathrm{NaOCl}$ treatment $(p<0.05)$. Regardless of the concentration, $\mathrm{NaOCl}$ had significantly higher efficiency in eliminating $E$. faecalis when it was used with DNase $(p<0.05)$. Furthermore, $5 \% \mathrm{NaOCl}$ exhibited a higher bactericidal effect against E. faecalis than $0.5 \%$ with or without DNase. However, $0.5 \% \mathrm{NaOCl}$ with DNase showed similar efficacy to $5 \%$ $\mathrm{NaOCl}$ without DNase $(p>0.05)$.

\section{Discussion}

eDNA is an important component in the extracellular matrix of $E$. faecalis biofilms. ${ }^{5,6}$ Researchers have investigated the function of eDNA to control biofilm formation since bacterial biofilms are related to persistent infections and provide strong bacterial resistance against antimicrobial agents. ${ }^{21-25} E$. faecalis is commonly isolated from failed root canal systems due to its ability to survive conventional endodontic treatment and periods of nutrient limitation and other challenging growth conditions. 3,26 The sensitivity of $E$. faecalis biofilms - particularly early in development - to DNase has been previously reported. ${ }^{5,27}$ We hypothesized that a clinical model of EF biofilm formation would show decreased biomass by combining eDNA reduction and $\mathrm{NaOCl}$ treatment.

First, we investigated the effects of eDNA inhibition on the growth of $E$. faecalis per se and its biofilm formation. With DNase, the inhibitor of eDNA used in this clinical model, it was important to determine the duration of its application into the infected root canal system. For this end, we cultured E. faecalis with DNase for 2 days or treated the biofilms that had formed for 2 days with DNase for 1 hour. The results showed that $E$. faecalis and its biofilm, as well as eDNA, were removed more effectively when cultured in the presence of DNase when compared to treatment with DNase after biofilm formation (Figure $1 b-d$ ). A recent study reported that DNase treatment does not disperse 24-hour matured $E$. faecalis biofilms formed in root canal systems. ${ }^{28}$ Furthermore, some studies have demonstrated that eDNA played an important role in the early stage of biofilm formation. ${ }^{6,10}$ Our results are consistent with a model in which early exposure of DNase to the biofilm may be more effective for $E$. faecalis biofilm formation inhibition when compared to the short-term treatment of DNase to matured biofilm.

Next, we identified the structure of the $E$. faecalis biofilm by CLSM and SEM according to DNase treatment. We detected exopolysaccharides surrounding bacterial cells in situ using CLSM to further confirm the potential of biofilm formation in $E$. faecalis. The results showed that the DNase-treated groups exhibited a significantly lower exopolysaccharides volume when compared to the control group (Figure 2a-d). Moreover, the exopolysaccharides volume of the DNase-2d group was significantly lower than that of the DNase-1h group. In fact, polysaccharides are believed to be a major prerequisite for biofilm formation. ${ }^{29}$ Notably, there have been several studies showing the existence of a correlation between eDNA and exopolysaccharides. ${ }^{30-32}$ In this regard, the dimensional evaluation of exopolysaccharides may reflect the amount and integrity of the biofilm. Furthermore, this evaluation can be an alternative method for the crystal violet staining. The staining quantification is known to be less useful in determining clinically-relevant biofilm factors, although being 0 generally used to evaluate biofilm biomass in toto. ${ }^{33}$ In SEM observation, the microstructure of DNase-treated biofilms exhibited more disintegrated characteristics when compared to the control (Figure 3). Previous studies demonstrated that eDNA was required to stabilize the biofilm structure, and the addition of exogenous DNases could prove a potent strategy for controlling biofilm growth.7,34 Therefore, it can be suggested that DNase might hamper biofilm formation by producing structure defects in the extracellular matrix. Corroborating previous studies, our results obtained by CLSM and SEM indicate that eDNA removal may interfere with the structural integrity of the nascent biofilm.

Lastly, we investigated the effect of eDNA removal from the biofilm on the susceptibility of $E$. faecalis to $\mathrm{NaOCl} . \mathrm{NaOCl}$ is the most widely used material to irrigate in dental procedures due to its high antimicrobial activity and capacity to dissolve organic tissue. ${ }^{35}$ However, $\mathrm{NaOCl}$ has drawbacks including an unpleasant odor and high toxicity that induces irritation when in contact with surrounding tissue. ${ }^{36}$ Therefore, in an attempt to reduce the $\mathrm{NaOCl}$ concentration, we used DNase and investigated whether DNase increases the efficacy of $\mathrm{NaOCl}$ against $E$. faecalis 
in biofilms. According to the results of this study, $5 \% \mathrm{NaOCl}$ exhibited a higher antibacterial effect against $E$. faecalis than $0.5 \%$ with or without DNase. However, the gap in elimination efficiency between $5 \%$ and $0.5 \% \mathrm{NaOCl}$ became smaller when DNase was used, mainly for potentiation of the effect of the low $\mathrm{NaOCl}$ concentration. Notably, $0.5 \% \mathrm{NaOCl}$ combined with DNase exhibited statistically similar values to $5 \% \mathrm{NaOCl}$ without DNase, suggesting that DNase adjunctive treatment could represent a useful strategy for improving antimicrobial action while reducing $\mathrm{NaOCl}$ concentration.

\section{Conclusions}

The results suggest that inhibition of eDNA leads to decrease of $E$. faecalis biofilm formation and increase E. faecalis susceptibility to $\mathrm{NaOCl}$ especially at low concentration $(0.5 \%)$. Therefore, our results suggest that inhibition of eDNA would be beneficial for removal of $E$. faecalis biofilm, especially by facilitating the efficacy of $\mathrm{NaOCl}$ and reducing its concentration for safer clinical use.

\section{Acknowledgements}

The study was supported by the Biomedical Research Institute, Chonbuk National University Hospital in 2017, and a grant of the Korea Health Technology R\&D Project through the Korea Health Industry Development Institute (KHIDI), funded by the Ministry of Health \& Welfare, Republic of Korea (grant number: HI18C0432).

Mi-Kyung Yu and Mi-Ah Kim contributed equally to this work as first authors.

Won-Jun Sohn and Kyung-San Min contributed to this work as co-corresponding authors

\section{References}

1- Siqueira JF Jr, Rôças IN. Clinical implications and microbiology of bacterial persistence after treatment procedures. J Endod. 2008;34(11):1291-301.

2- Zhu X, Wang Q, Zhang C, Cheung GS, Shen Y. Prevalence, phenotype, and genotype of Enterococcus faecalis isolated from saliva and root canals in patients with persistent apical periodontitis. J Endod. 2010;36(12):1950-5

3- Stuart CH, Schwartz SA, Beeson TJ, Owatz CB. Enterococcus faecalis: its role in root canal treatment failure and current concepts in retreatment. J Endod. 2006;32(2):93-8.
4- Figdor D, Davies JK, Sundqvist G. Starvation survival, growth and recovery of Enterococcus faecalis in human serum. Oral Microbiol Immunol. 2003;18(4):234-9.

5- Whitchurch CB, Tolker-Nielsen T, Ragas PC, Mattick JS. Extracellular DNA required for bacterial biofilm formation. Science. 2002;295(5559):1487.

6- Barnes AM, Ballering KS, Leibman RS, Wells CL, Dunny GM. Enterococcus faecalis produces abundant extracellular structures containing DNA in the absence of cell lysis during early biofilm formation. MBio. 2012;3(4):e00193-12.

7- Molin S, Tolker-Nielsen T. Gene transfer occurs with enhanced efficiency in biofilms and induces enhanced stabilisation of the biofilm structure. Curr Opin Biotechnol. 2003;14(3):255-61.

8- Thomas VC, Hiromasa Y, Harms N, Thurlow L, Tomich J, Hancock LE. A fratricidal mechanism is responsible for eDNA release and contributes to biofilm development of Enterococcus faecalis. Mol Microbiol. 2009;72(4):1022-36.

9- Badel S, Laroche C, Gardarin C, Bernardi T, Michaud P. New method showing the influence of matrix components in Leuconostoc mesenteroides biofilm formation. Appl Biochem Biotechnol. 2008; 151(2-3):364-70.

10- Thomas VC, Thurlow LR, Boyle D, Hancock LE. Regulation of autolysis-dependent extracellular DNA release by Enterococcus faecalis extracellular proteases influences biofilm development. J Bacteriol. 2008;190(16):5690-8.

11- Das T, Sharma PK, Busscher HJ, van der Mei HC, Krom BP. Role of extracellular DNA in initial bacterial adhesion and surface aggregation. Appl Environ Mcrobiol. 2010;76(10):3405-8.

12- Bolfoni MR, Ferla MS, Sposito OS, Giardino L, Jacinto RC, Pappen FG. Effect of a surfactant on the antimicrobial activity of sodium hypochlorite solutions. Braz Dent J. 2014;25(5):416-9.

13- Spencer HR, Ike V, Brennan PA. Review: the use of sodium hypochlorite in endodontics: potential complications and their management. Br Dent J. 2007;202(9):555-9.

14- Pashley EL, Birdsong NL, Bowman K, Pashley DH. Cytotoxic effects of $\mathrm{NaOCl}$ on vital tissue. J Endod. 1985;11(12):525-8.

15- Cunningham WT, Balekjian AY. Effect of temperature on collagendissolving ability of sodium hypochlorite endodontic irrigant. Oral Surg Oral Med Oral Pathol. 1980;49(2):175-7.

16- Abou-Rass M, Oglesby SW. The effects of temperature, concentration, and tissue type on the solvent ability of sodium hypochlorite. J Endod. 1981;7(8):376-7.

17- Hecker S, Hiller KA, Galler KM, Erb S, Mader T, Schmalz G. Establishment of an optimized ex vivo system for artificial root canal infection evaluated by use of sodium hypochlorite and the photodynamic therapy. Int Endod J. 2013;46(5):449-57.

18- Wang Y, Xiao S, Ma D, Huang X, Cai Z. Minimizing concentration of sodium hypochlorite in root canal irrigation by combination of ultrasonic irrigation with photodynamic treatment. Photochem Photobiol. 2015;91(4):937-41.

19- Yanling C, Hongyan L, Xi W, Wim C, Dongmei D. Efficacy of relacin combined with sodium hypochlorite against Enterococcus faecalis biofilms. J Appl Oral Sci. 2018;26:e20160608.

20- Heydorn A, Nielsen AT, Hentzer M, Sternberg C, Givskov M, Ersbøll $B K$, et al. Quantification of biofilm structures by the novel computer program COMSTAT. Microbiology. 2000;146(Pt 10):2395-407. 21- Brady RA, Leid JG, Calhoun JH, Costerton JW, Shirtliff ME. Osteomyelitis and the role of biofilms in chronic infection. FEMS Immunol Med Microbiol. 2008;52(1):13-22.

22- Costerton JW, Stewart PS, Greenberg EP. Bacterial biofilms: a common cause of persistent infections. Science. 1999;284(5418):131822.

23- Stewart PS, Costerton JW. Antibiotic resistance of bacteria in biofilms. Lancet. 2001;358(9276):135-8. 
24- Simoes M. Antimicrobial strategies effective against infectious bacterial biofilms. Curr Med Chem. 2011;18(14):2129-45.

25- Okshevsky M, Regina VR, Meyer RL. Extracellular DNA as a target for biofilm control. Curr Opin Biotechnol. 2015;33:73-80.

26- Hancock HH $3^{\text {rd }}$, Sigurdsson A, Trope M, Moiseiwitsch J. Bacteria isolated after unsuccessful endodontic treatment in a North American population. Oral Surg Oral Med Oral Pathol Oral Radiol Endod. 2001;91(5):579-86

27- Guiton PS, Hung CS, Kline KA, Roth R, Kau AL, Hayes E, et al. Contribution of autolysin and sortase a during Enterococcus faecalis DNA-dependent biofilm development. Infec Immun. 2009;77(9):362638.

28- Schlafer S, Garcia J, Meyer RL, Vaethd M, Neuhause KW. Effect of DNase treatment on adhesion and early biofilm formation of Enterococcus faecalis. Eur Endod J. 2018;3:8206.

29- Camporese A, Tizianel G, Bruschetta G, Cruciatti B, Pomes A. Human meningitis caused by Streptococcus suis: the first case report from north-eastern Italy. Infez Med. 2007;15(2):111-4.

30- Ghafoor A, Hay ID, Rehm BH. Role of exopolysaccharides in Pseudomonas aeruginosa biofilm formation and architecture. Appl Environ Microbiol. 2011;77(15):5238-46.
31- Castillo Pedraza MC, Novais TF, Faustoferri RC, Quivey RG Jr, Terekhov A, Hamaker BR, et al. Extracellular DNA and lipoteichoic acids interact with exopolysaccharides in the extracellular matrix of Streptococcus mutans biofilms. Biofouling. 2017;33(9):722-40.

32- Wang S, Liu X, Liu H, Zhang L, Guo Y, Yu S, et al. The exopolysaccharide Psl-eDNA interaction enables the formation of a biofilm skeleton in Pseudomonas aeruginosa. Environ Microbiol Rep. $2015 ; 7(2): 330-40$.

33- Pitts B, Hamilton MA, Zelver N, Stewart PS. A microtiter-plate screening method for biofilm disinfection and removal. J Microbiol Methods. 2003;54(2):269-76.

34- Jakubovics NS, Shields RC, Rajarajan N, Burgess JG. Life after death: the critical role of extracellular DNA in microbial biofilms. Lett Appl Microbiol. 2013;57(6):467-75.

35- Zehnder M. Root canal irrigants. J Endod. 2006;32(5):389-98.

36- Mohammadi Z. Sodium hypochlorite in endodontics: an update review. Int Dent J. 2008;58(6):329-41. 